\title{
Research of the Penetration Process for Concrete Target Based on the Finite Element Method
}

\author{
WANG Feng ${ }^{a}$, DAI Jun-tao ${ }^{b}$, Gao Bin-sen $^{c}$, Ma Yi-hong $^{d}$ \\ College of Civil and environmental Engineering, Anhui Xinhua University, Hefei, 230088, Anhui, China



Keywords: finite element method (FEM); concrete; residual velocity; numerical simulation; penetration Abstract: A damage rate dependent constitutive model suitable for penetration calculation is introduced in this paper. The finite element program is applied to simulate the penetration of a projectile into concrete target. The simulation results of residual velocity are in good agreement with the experimental results, which can simulate the pits and damage distributions of concrete projectiles penetrating into concrete targets, and the calculation results for oblique penetration are also analyzed. It shows the rationality and effectiveness of the method and program established in this paper, and it provides an important alternative for numerical analysis of concrete penetration process.

\section{Introduction}

Concrete is a special composite material. It has many advantages, such as good formability, water resistance, fire resistance, durability, raw materials, and low cost. It has been widely applied not only in civil buildings, but also in military facilities and protection works. It is a desire to analyze the penetration mechanism and penetration effect of projectiles to concrete in theory. However, concrete is a brittle porous material inhomogeneous and anisotropic, its structure is more complex, in the dynamic loading case, and show the characteristics of strain rate, the dynamic process of missile penetration contains large deformation, damage and failure, damage and damage of the materials is very complex the theoretical analysis is very difficult. In the current research methods, field experimental research is essential, but it is costly and time-consuming.

Tate $\mathrm{A}[1]$, Anderson C E and Walker J D[2] are committed to building simplified analytical models. However, simplified analytical models often have too many physical assumptions and deviate from practical ones. The numerical basis for the penetration problem of concrete is a very effective method in the simulation method of finite element and finite difference, precision and reliability of both basic equation, in the physical model of the solution and results, it will be much better than the approximate analysis, and cost less. Therefore, it is of great practical significance to carry out the numerical simulation of the concrete problem.

\section{Calculation method}

The basic equations given by the conservation theorem of continuum mechanics can be expressed in tensor form.

$$
\begin{array}{lr}
\text { Mass conservation } & \tilde{\alpha}+\rho \nabla \cdot \tilde{v}=0 . \\
\text { Conservation of momentum } & \rho \tilde{\sigma}=\nabla \cdot \tilde{\sigma}+\tilde{b} . \\
\text { Conservation of energy } & E^{\alpha}=\tilde{\sigma}: \nabla \tilde{v} .
\end{array}
$$

where $\tilde{v}$ is the particle velocity, $\tilde{\sigma}$ is Cauchy stress tensor, $\tilde{b}$ is unit volume physical strength, $E$ is internal energy of unit volume, $\nabla$ is Hamilton operator, “"” is derivative of following body. 
When the Lagrange method is used for the finite element discretization of the above basic equations, the mass conservation equation is automatically satisfied. The energy equation can be expressed as follows.

$$
E_{0}=-P \&_{+} V S_{i j} \& \text {. }
$$

where $P$ is hydrostatic pressure, $V$ is specific volume, $S_{i j}$ is partial stress tensor, $e_{i j}$ is partial strain tensor. The formula (4) has no spatial partial derivative, so it can be calculated directly by increment. So the equation of motion is the only conservation equation that needs to be discretized by finite element. After the centralized mass method is used, the motion equation is discrete to the mass motion equation expressed by the force of the node.

$$
m_{I} \Downarrow_{I}^{\&}=\tilde{f}_{I}^{e x t}-\tilde{f}_{I}^{\mathrm{int}} .
$$

where $m_{I}$ is centralized quality of node $I, \tilde{f}_{I}^{\text {ext }}(t)=\int_{\Omega e} \tilde{N}^{T}(\tilde{x}) \tilde{b}(\tilde{x}, t) d \Omega+\int_{\Omega e} \tilde{N}^{T}(\tilde{x}) \tilde{t}_{n}(\tilde{x}, t) d \Omega$ is node external force, $\tilde{b}$ and $\tilde{t}_{n}$ are the strength and surface force on the unit $\Omega_{e}, \tilde{f}_{I}^{\text {int }}(t)=\int_{\Omega e} \widetilde{B}^{T}(\tilde{x}) \tilde{\sigma}(\tilde{x}, t) d \Omega$ is nodal force.

The time integration of formula (5) will get node speed, and get the unit strain rate and the corresponding strain increment through geometric relation. Then we can get all physical quantities such as stress tensor, internal energy and density by mass conservation, energy conservation and constitutive equation.

\section{Material model}

The projectile used in the calculation is a steel rod projectile, and the material is taken as a linear hardened body. The static water pressure and the volume deformation are described by the following Gruneisen equation of state[3].

$$
P=\left(K_{1} \mu+K_{2} \mu^{2}+K_{3} \mu^{3}\right)(1-0.5 \gamma \mu)+\gamma \rho E .
$$

where $\mu=V_{0} / V-1$ is compression ratio, $\gamma$ is Gruneisen parameters, $E$ is specific internal energy.

The stress deviation obeys the incremental elastoplastic stress-strain relation, that is

$$
d S_{i j}=2 G\left(d e_{i j}-d e_{i j}^{p}\right) \text {. }
$$

The material obeys the Mises yield criterion, and the yield strength is taken as a linear hardening function.

$$
Y=Y_{0}+\left(Y_{u}-Y_{0}\right) \bar{\varepsilon}_{P} / \bar{\varepsilon}_{u} .
$$

\begin{tabular}{|c|c|c|c|c|c|c|c|c|}
\hline \multirow{2}{*}{$\begin{array}{c}\rho \\
\left(\mathrm{kg} / \mathrm{m}^{3}\right)\end{array}$} & \multirow{2}{*}{$\begin{array}{c}\mathrm{G} \\
(\mathrm{Gpa})\end{array}$} & \multirow{2}{*}{$\begin{array}{c}\mathrm{Y}_{0} \\
\text { (Gpa) }\end{array}$} & \multirow{2}{*}{$\begin{array}{c}\mathrm{Y}_{\mathrm{u}} \\
(\mathrm{Gpa})\end{array}$} & \multirow{2}{*}{$\bar{\varepsilon}_{u}$} & \multicolumn{3}{|c|}{$\begin{array}{l}\text { Parameter of state } \\
\text { equation (Gpa) }\end{array}$} & \multirow[t]{2}{*}{$\gamma$} \\
\hline & & & & & $\mathrm{K}_{1}$ & $\mathrm{~K}_{2}$ & $\mathrm{~K}_{3}$ & \\
\hline 7823 & 77.5 & 0.355 & 0.60 & 1.00 & 164 & 294 & 500 & 2.0 \\
\hline
\end{tabular}

where $Y_{0}$ is initial yield stress, $Y_{u}$ is ultimate yield stress, $\bar{\varepsilon}_{P}$ is equivalent plastic strain, $\bar{\varepsilon}_{u}$ is ultimate equivalent plastic strain.

The relevant physical parameters and calculation parameters used in the calculation are shown in Tab. 1.

Tab.1. Material parameters of a projectile

In view of the physical problems calculated in this paper, we adopt the following form of damage rate related constitutive model for concrete[4 5].

Considering the pressure dependence and strain rate effect of material strength, the equivalent strength of the normalization can be expressed as

$$
\sigma^{*}=\left[A(1-D)+B *^{*^{N}}\right](1+C \ln \&) .
$$

where $\mathrm{D}$ is damage amount $(0 \leq \mathrm{D} \leq 1.0), p^{*}=p / f_{c}$ is normalized pressure, $p$ is hydrostatic pressure, $f_{\mathrm{c}}$ is quasi-static uniaxial compression strength, $\&=\& \& \&$ is equal effect variability, $\&=1.0(\mathrm{sec})^{-1}$ is reference strain rate, $\mathrm{A}, \mathrm{B}, \mathrm{C}$, and $\mathrm{N}$ are material parameters.

Concrete is a porous brittle material, and its failure is characterized by a large number of microcracks in the loading process, resulting in the change of the microstructure of materials, resulting in the inoculation, expansion and convergence of micro defects, leading to macroscopic cracking or material failure. For the 
concrete targets in the high-speed penetration process, we assume that the damage of the material is completely caused by the plastic flow deformation, and the damage evolution equation can be expressed as

$$
\hat{\&}=\frac{\bar{\varepsilon}_{p}^{\mathbb{E}}+\varepsilon_{v}}{\bar{\varepsilon}_{p}^{f}} \text {. }
$$

where $\bar{\varepsilon}_{p}$ is equivalent plastic strain, $\varepsilon_{v}^{p}$ is plastic volume deformation caused by static water pressure correlation in strength model, $\bar{\varepsilon}_{p}^{f}$ is ultimate equivalent plastic strain.

In the program calculation, the material of the target plate is taken as the concrete material of the compressive strength $f_{\mathrm{c}}=48 \mathrm{MPa}$, the density $\rho_{0}=2440 \mathrm{~kg} / \mathrm{m}^{3}$, the shear modulus $\mathrm{G}=16.86 \mathrm{GPa}$

\section{Numerical simulation calculation and result analysis}

In this paper, a three-dimensional numerical simulation of a steel rod projectile penetrated into a concrete target is carried out by using the HVP-3D program and the above material model.

Hanchak S.J. et al. [6] has carried out a series of experimental studies on the high speed concrete target of oval rod projectile, and obtained a lot of valuable experimental data. For this reason, we first simulated their experimental results to verify the reasonableness of the model. The projectile used by the Hanchak S.J. et al. Experiment is a steel bar projectile with a tangent oval warhead, as shown in Fig.1. The thickness of the concrete target is $178 \mathrm{~mm}$. Because of the symmetry of the structure and loading mode of the rod and the target plate, the $1 / 2$ structure is only needed to be modeled. The eight node hexahedral element is used to mesh the projectile and the target plate. In order to improve the computational efficiency and ensure the accuracy of the calculation, the target plate grid is set into a variable grid. A fine grid is taken near the penetration channel and a coarse mesh in the distance. The initial grid distribution of the projectile and the target is shown in Fig.2.

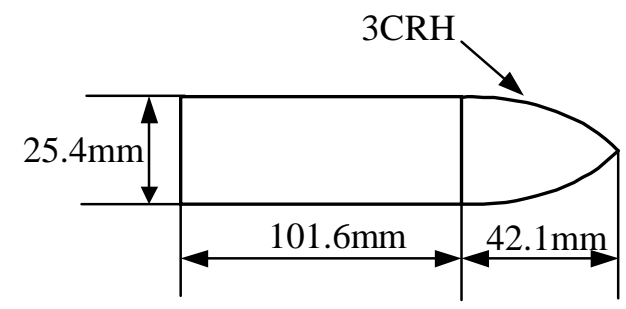

Fig.1. Projectile structure $(0.5 \mathrm{~kg})$

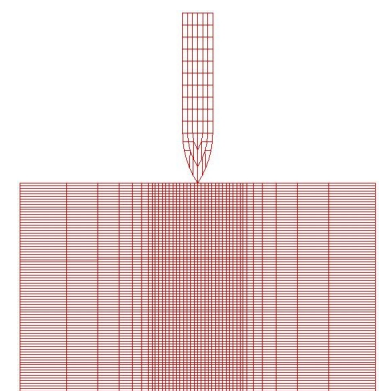

Fig.2. The initial time grid distribution map of an oval rod projectile penetrated into the target plate

Fig.3 shows the comparison between the experimental values of the residual speed $V_{r}$ of the oval shaped rod projectile penetrating the concrete target with different initial velocity $\mathrm{V}_{0}$ and the simulation results, which shows that both are in good agreement, which shows the rationality and validity of the model and the program established in this paper.



Fig.3. Comparison between experimental and simulated results of residual velocity



Fig.4. The relationship between the shape of the warhead and the penetration depth

Fig. 4 is the relationship between the penetration depth $\mathrm{H}$ of the projectile and the initial velocity $\mathrm{V}_{0}$ when the warhead is conical, oval and spherical. The size of the oval rod is the same as that of the projectile, 
and the diameter of the projectile is the same as the length of the rod. The height of the cone-shaped warhead is the same as that of the egg shaped warhead. It can be seen from the diagram that, under the same conditions, the penetration ability of the cone rod is the strongest, and the penetration ability of the spherical rod is the weakest. This is because the sharper the shape of the warhead is, the smaller the penetration resistance is, and the deeper the penetration is. The more flat the warhead is, the larger the penetration of the warhead will be, and the deeper the penetration will be.

Fig.5 is the stereogram and symmetry section of an oval shaped bar projectile when the initial velocity $746 \mathrm{~m} / \mathrm{s}$ is penetrated into the concrete target. The results of the calculation well simulate the pit formation and damage distribution of the rod projectile penetrated into the concrete target. Concrete target damage near the warhead at the most serious, the body and the strength of the target material is large, both experiments and calculations show that rod projectile penetrating a target plate, not mass wasting, almost no deformation. Damage behavior of the unit in the penetration process is described by the amount of damage $\mathrm{D}$ and equivalent plastic strain criterion, once the damage amount of $\mathrm{D}$ is larger than 1 or the equivalent plastic strain $\bar{\varepsilon}_{u}$ exceeds the maximum allowed unit is regarded as a failure, its strength is set to zero. The mesh distortion of the destruction unit is very large, and it is not plotted in the clear drawing. In fact, most of the blank parts between the targets are destroyed units.

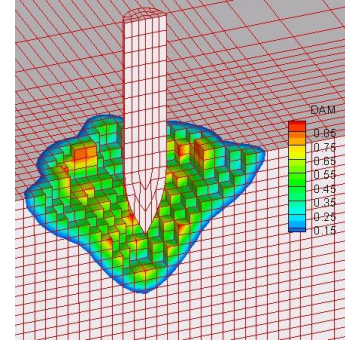

(a) $\mathrm{t}=37.8 \mu \mathrm{s}$

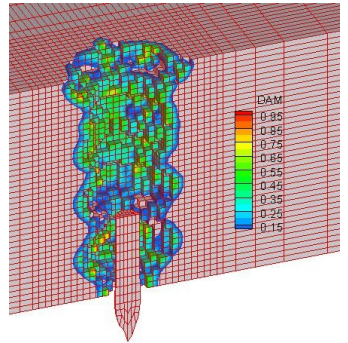

(b) $\mathrm{t}=337.5 \mu \mathrm{s}$

Fig.5. Damage distribution of the initial velocity $746 \mathrm{~m} / \mathrm{s}$ rod projectile penetrating the concrete target at different time

\section{Conclusions}

In this paper, a concrete material model suitable for 3D impact mechanics numerical simulation is introduced, and a series of numerical simulation is carried out for 3D projectile penetrating into concrete target by three-dimensional finite element program. The results of the penetration are in good agreement with the experimental results, and the results of the oblique penetration are also analyzed. This indicates that the method and program established in this paper are reasonable and effective, and it provides an important alternative for numerical analysis of concrete penetration process.

\section{References}

[1] Tate A. A theory for the deceleration of long rod after impact[J]. J Mech Phys Solids, 1967,15:387-399.

[2] Anderson C E, Walker J D. An examination of long-rod penetration[J]. Int J.Impact Eng, 1991,11:481-501.

[3] J.R. Robbins, J.L. Ding, Y.M. Gupta. Load spreading and penetration resistance of layered structures-a numerical study[J]. Int J.Impact Eng, 2004,30(6):593 - 615.

[4] Chang jiang H E, Zhi lu Y U, Fan Z B. An Eulerian Numerical Method on Dynamic Impact Problem of Concrete[J]. Explosion \& Shock Waves, 2002. (in Chinese)

[5] Zhang F G, Li E Z. Computational model for concrete subjected to large strains, high strain rates, and high pressures[J]. Explosion \& Shock Waves, 2002. (in Chinese)

[6] Hanchak S J, Forrestal M J, Young E R, et al. Perforation of Concrete Slabs with 48MPa and 140Mpa Unconfined Compressive Strengths[J]. Int J.Impact Eng, 1992 ,12(1):127. 